\title{
Transition operators entering neutrinoless double electron capture to excited nuclear states
}

\author{
J. D. Vergados* \\ Max Planck für Kernphysik, Saupfercheckweg 1, DE-69120 Heidelberg, Germany and \\ CERN Theory Division, Geneva, Switzerland
}

(Received 17 July 2011; published 28 October 2011)

\begin{abstract}
We construct the effective transition operators relevant for neutrinoless double electron capture leading to final nuclear states different than $0^{+}$. From the structure of these operators we see that if such a process is observed experimentally, it will be very helpful in singling out the very important light neutrino mass contribution from the other lepton violating mechanisms.
\end{abstract}

DOI: 10.1103/PhysRevC.84.044328

PACS number(s): 21.60.Cs, 23.40.Bw, 25.30.Pt, 13.15.+g

\section{INTRODUCTION}

The nuclear double $\beta$ decay is a process that was considered a very long time ago [1]. It can occur whenever the ordinary (single) $\beta$ decay is forbidden due to energy conservation or greatly suppressed due to angular momentum mismatch. It could proceed with the emission of two neutrinos or in a neutrinoless mode. The $2 \nu \beta \beta$ decay is just an example of a second-order weak interaction. The exotic neutrinoless double $\beta$ decay ( $0 \nu \beta \beta$ decay) is the most interesting since it violates lepton number by two units. It was first considered by Furry [2] more than half a century ago as soon it was realized that the neutrino might be a Majorana particle. Today, 70 years later, $0 \nu \beta \beta$ decay, i.e.,

$$
(A, Z) \rightarrow(A, Z+2)+e^{-}+e^{-}, \quad(0 \nu \beta \beta \text { decay }),
$$

with $\Delta=Q$ the available energy, continues to be one of the most interesting processes for the following reasons: (i) It will establish whether lepton violating processes exist in the universe. (ii) It is perhaps the only process that can reveal whether neutrinos are Majorana particles, i.e., the particle coincides with its charge conjugate, or Dirac particles, if it does not. (iii) It will aid in settling the outstanding question of the absolute scale of the neutrino masses, especially if this scale turns out to be very small. Neutrino oscillation experiments can only determine the mass squared differences. For recent reviews, see Refs. [3-6].

If this process is allowed, other related processes in which the charge of the nucleus is decreased by two units may also occur, if they happen to be allowed by energy and angular momentum conservation laws, e.g.,

$$
(A, Z) \rightarrow(A, Z-2)+e^{+}+e^{+},
$$

$\Delta=Q-4 m_{e} c^{2}(0 v$ positron emission),

$$
(A, Z)+e^{-} \rightarrow(A, Z-2)+e^{+},
$$

$\Delta=Q-2 m_{e} c^{2}(0 v$ electron positron conversion),

$$
(A, Z)+e^{-}+e^{-} \rightarrow(A, Z-2)+\text { x rays },
$$

$\Delta=Q(0 v$ double electron capture $)$.

\footnotetext{
*Vergados@cc.uoi.gr; Permanent address: Theoretical Physics Division, University of Ioannina, Ioannina, GR-451 10, Greece.
}

The last process is always possible whenever Eq. (3) is. It was first considered long ago [7,8] as a two-step process: In the first step the two neutral atoms $(A, Z)$ and the excited atom $(A, Z-2)$ get admixed via the lepton number violating interaction. In the second step the $(A, Z-2)$ atom deexcites, emitting two hard $\mathrm{x}$ rays; and the nucleus, if it is found in an excited state, deexcites, emitting $\gamma$ rays. The lifetime expected is very long, since the above mixing amplitude is tiny compared to the energy difference of the two atoms involved. It has recently, however, been gaining in importance [9-12] after ion Penning traps [13] made it possible to accurately determine the $Q$ values, which gave rise to the presence of resonances, which in turn could lead to an increase of the width by many orders of magnitude [9]. Decays to excited states are in some cases possible and provide additional experimental information, e.g., $\gamma$ rays following their deexcitation.

Furthermore neutrinoless double electron capture is of experimental interest, since one expects this reaction to be essentially free of the two neutrino background, which plagues ordinary neutrinoless double $\beta$ decay. It has also recently been realized [10] that the resonance condition can be fulfilled for transitions to excited states. Thus, e.g., in the case of ${ }^{156} \mathrm{Dy} \rightarrow{ }^{156} \mathrm{Gd}$, such excited states can be $0^{-}, 1^{-}, 0^{+}, 1^{+}$, and $2^{+}$.

Before proceeding further in examining neutrinoless double electron capture, we note the following:

(i) Transitions to $0^{-}, 1^{-}$states, to the best of our knowledge, have not been considered previously, since they are energetically forbidden in double $\beta$ decay. Such transitions can naturally occur, however, in the presence of right-handed currents, arising by interference between the left- and righthanded currents, i.e., of the type $j_{L} \times j_{R}$, with an amplitude essentially independent of the neutrino mass. We will show that this process can occur in double electron capture, even though the electrons are bound.

(ii) Transitions to $0^{+}$states, both the ground states and excited states, can occur both in the mass mechanism and the $j_{L} \times j_{R}$. The latter contribution in ordinary double $\beta$ decay occurs since the produced electrons have sufficiently high momenta. In double electron capture, instead of the electron momentum one can exploit the nuclear recoil term.

(iii) Transitions to $1^{+}$and $2^{+}$can occur only if the electron momentum is sufficiently high, as in neutrinoless double $\beta$ 
decay, or by retaining the nuclear recoil term in the hadronic current. In double electron capture this can naturally happen if one of the electrons is captured from a $p$ orbit.

In the present paper we will derive the effective transition operators relevant for neutrinoless double electron capture, emphasizing the mode which is independent of the neutrino mass. The neutrino mass contribution has been previously examined, see, e.g., the recent work in Ref. [9] and references therein.

\section{NUCLEAR TRANSITION OPERATORS LEADING TO NEGATIVE PARITY STATES}

To leading order, such transitions cannot occur in a neutrino mass mechanism. In the $j_{L} \times j_{R}$ case, however, the chiralities involved are such that in the intermediate neutrino propagator one picks the momentum, not the mass. The neutrino momentum leads to the following:

(i) The time component of the momentum leads to an average energy difference $E_{n, 1}-E_{n, 2}$, where $E_{n, i}=\left\langle E_{n}\right\rangle-$ $M_{i}+E_{e}(i)$, with $E_{e}(i)$ the electron energy, $i=1,2$. This can lead to positive parity transitions in double $\beta$ decay, but it is not the dominant contribution.

(ii) The space part of the momentum leads to an effective operator which is proportional to $\hat{r} \frac{d}{d r} \frac{1}{r}$ with $\mathbf{r}=\mathbf{r}_{1}-\mathbf{r}_{2}, r=$ $\left|\mathbf{r}_{1}-\mathbf{r}_{2}\right|$. This naturally leads to negative parity transitions.

Positive parity transitions can, of course, occur in neutrinoless double $\beta$ decay via this mechanism by retaining the dipole term in the expansion of the essentially distorted plane wave function of the two electrons, which leads to the term $i\left(\mathbf{p}_{1} \cdot \mathbf{r}_{1}+\mathbf{p}_{2} \cdot \mathbf{r}_{2}\right) \hat{r} \frac{d}{d r} \frac{1}{r}$. This possibility does not exist in neutrinoless double electron capture. We will, however, examine alternative possibilities in the next section.

The effective lepton violating transition operator, within the closure approximation over the intermediate states, which holds for neutrinoless double $\beta$ decay and is expected to be even better in neutrinoless double electron capture, can be cast in the form

$$
\begin{aligned}
\mathcal{M}= & \frac{1}{\sqrt{2}}\left(\frac{G_{F}}{\sqrt{2}}\right)^{2} 2 \frac{2 i}{8 \pi R_{0}^{2}} \lambda u_{\mu \nu k}^{R} \\
& \times \sum_{n, m} \phi_{n_{1}, 0}\left(r_{n}\right) \phi_{n_{2}, 0}\left(r_{m}\right) J_{R}^{\mu}(n) J_{L}^{\nu}(m) \hat{r}_{n, m}^{k} f\left(x_{n, m}\right) \\
& +L \leftrightarrow R
\end{aligned}
$$

with $\lambda$ the lepton violating parameter [3], $\phi_{n_{1}, 0}\left(r_{n}\right) \phi_{n_{2}, 0}\left(r_{m}\right)$ $\left(r_{n}=r_{1}, r_{m}=r_{2}\right)$ the bound $s$-electron wave functions, and

$$
\begin{aligned}
u_{\mu \nu k}^{R} & =\bar{u}\left(p_{1}\right) \gamma_{\mu} \gamma_{k} \gamma_{\nu}\left(1-\gamma_{5}\right) u^{c}\left(p_{2}\right), \\
u_{\mu \nu k}^{L} & =\bar{u}\left(p_{1}\right) \gamma_{\mu} \gamma_{k} \gamma_{\nu}\left(1+\gamma_{5}\right) u^{c}\left(p_{2}\right), \\
J_{L}^{\mu}(n) & =\tau_{-}(n)\left(g_{V},-g_{A} \sigma_{n}\right), \\
J_{R}^{v}(m) & =\tau_{-}(m)\left(g_{V},+g_{A} \sigma_{m}\right), \\
f\left(x_{n, m}\right) & =\frac{d}{d x} \frac{1}{x} J\left(\delta_{0}\right), \\
x & =x(n, m)=\left|\mathbf{r}_{n}-\mathbf{r}_{m}\right| / R_{0}, \\
J\left(\delta_{0}\right) & =\frac{2}{\pi} \int_{0}^{\infty} \frac{\sin y}{y+\delta_{0}} d y,
\end{aligned}
$$

$$
\delta_{0}=\left(\left\langle E_{n}\right\rangle-M_{i}\right)\left|\mathbf{r}_{n}-\mathbf{r}_{m}\right| ;
$$

and in double electron capture,

$$
\delta_{0} \approx 0, \quad J\left(\delta_{0}\right) \approx 1, \quad f(x) \approx-\frac{1}{x^{2}} .
$$

The effective transition operator can be cast in the following forms:

(i) An operator associated with the time structure of the leptonic current:

$$
\ell_{0}=\bar{u}\left(p_{1}\right) \gamma_{0} \gamma_{5} u^{c}\left(p_{2}\right)
$$

which is

$$
\begin{aligned}
\tilde{\Omega}_{0}= & \lambda \frac{G_{F}^{2}}{2 \sqrt{2}} \frac{i}{\pi R_{0}^{2}} 4 g_{A}^{2} \\
& \times \sum_{n, m} \phi_{n_{1}, 0}\left(r_{n}\right) \phi_{n_{2}, 0}\left(r_{m}\right) \tau_{-}(n) \tau_{-}(m) \\
& \times \hat{r}_{n m} \cdot \omega_{0}(n, m) f\left(x_{n, m}\right) \\
\omega_{0}(n, m)= & i\left(\sigma_{n} \times \sigma_{m}\right)
\end{aligned}
$$

which can cause $0^{+} \rightarrow 0^{-}$transitions.

(ii) An operator associated with the space structure of the leptonic current:

$$
\vec{\ell}=\bar{u}\left(p_{1}\right) \vec{\gamma} u^{c}\left(p_{2}\right)
$$

that is,

$$
\begin{aligned}
\tilde{\Omega}_{1}= & \lambda \frac{G_{F}^{2}}{2 \sqrt{2}} \frac{i}{\pi R_{0}^{2}} g_{V} g_{A} \\
& \times \sum_{n, m} \phi_{n_{1}, 0}\left(r_{n}\right) \phi_{n_{2}, 0}\left(r_{m}\right) 2 \tau_{-}(n) \tau_{-}(m) \\
& \times\left[\hat{r}_{n m} \times \omega_{1}(n, m)\right] f\left(x_{n, m}\right), \\
\omega_{1}(n, m)= & \left(\sigma_{n}-\sigma_{m}\right),
\end{aligned}
$$

which can cause $0^{+} \rightarrow 1^{-}$transitions.

Since the $0 s$ electron wave functions vary slowly inside the nucleus, one can replace them by their average value and write:

$$
\tilde{\Omega}_{i}=\lambda \Lambda_{\mathrm{eff}}\left\langle(0 s)^{2} a_{B}^{3}\right\rangle \Omega_{i}, \quad \Lambda_{\mathrm{eff}}=\frac{G_{F}^{2}}{2 \sqrt{2}} \frac{i}{\pi R_{0}^{2} a_{B}^{3}},
$$

where the $\Lambda_{\text {eff }}$ has dimensions of energy and the average is over the radial part alone (the integral over the angles is unity). The dimensionless effective transition operators now become

$$
\begin{gathered}
\Omega_{0}=4 g_{A}^{2} \sum_{n, m} \tau_{-}(n) \tau_{-}(m) \hat{r}_{n m} \cdot \omega_{1}(n, m) f\left(x_{n, m}\right), \\
\omega_{0}(n, m)=i\left(\sigma_{n} \times \sigma_{m}\right), \\
\Omega_{1}=2 g_{V} g_{A} \sum_{n, m} \tau_{-}(n) \tau_{-}(m) \hat{r}_{n m} \times \omega_{1}(n, m) f\left(x_{n, m}\right), \\
\omega_{1}(n, m)=\sigma_{n}-\sigma_{m} .
\end{gathered}
$$

One can show that by averaging over the initial polarizations and summing over the final ones, one gets

$$
|M|^{2}=2 \sum_{i=0}^{1}\left|\left\langle f|| \Omega_{i}^{k}|| 0^{+}\right\rangle\right|^{2}
$$


with $k=i=J_{f}=$ rank of the operator (the factor of 2 comes from the leptonic current) and the double bar indicates its standard reduced matrix element $M$, which can cause $0^{+} \rightarrow$ $1^{-}$transitions. The mixing $V^{2}$ [9] between the two atoms takes the form

$$
V^{2}=|\lambda|^{2} \Lambda_{\text {eff }}^{2}\left[\left\langle(0 s)^{2} a_{B}^{3}\right\rangle\right]^{2}|M|^{2} .
$$

\section{TRANSITIONS TO POSITIVE PARITY STATES IN ELECTRON CAPTURE}

We will describe the transitions to positive parity states for two cases: one in which the captured electrons are in $s$ states and one in which an electron is in a $p$ orbit.

\section{A. Captured electrons are in s states}

As we have mentioned, the $0^{+} \rightarrow 0^{+}$transitions associated with double electron capture have been studied during the last few years. The contribution arising from the neutrino mass independent mechanism has not been studied, since the electron wave function cannot provide the extra parity change. This, however, can be accomplished via the recoil term in the nucleon current. ${ }^{1}$ In other words one of the hadronic currents is of the form discussed above, but the other is of the form [14]

$$
\begin{aligned}
J_{L}^{\mu}(n) & =\tau_{-}(n)\left(g_{V} \mathbf{D}_{n},-g_{A} C_{n}\right), \\
J_{R}^{v}(m) & =\tau_{-}(m)\left(g_{V} \mathbf{D}_{m},+g_{A} C_{m}\right),
\end{aligned}
$$

with

$$
\begin{aligned}
C_{n} & =\frac{1}{2 m_{N}} \sigma_{n} \cdot\left(\mathbf{q}_{n}-2 \mathbf{P}_{n}\right), \\
\mathbf{D} & =\frac{1}{2 m_{N}}\left[\left(\mathbf{q}_{n}-2 \mathbf{P}_{\mathbf{n}}\right) \times i \sigma_{n}\right] .
\end{aligned}
$$

Here $m_{N}$ and $\mathbf{P}_{n}$ are the nucleon mass and momentum of nucleon $n$, while $\mathbf{q}_{n}$ is the momentum transfer to that nucleon.

The procedure is a bit tedious but one can proceed as above to arrive at two operators:

(i) One operator associated with the leptonic current:

$$
\vec{\ell}=\bar{u}\left(p_{1}\right) \vec{\gamma} \gamma_{5} u^{c}\left(p_{2}\right),
$$

which is associated with an operator appropriate for $0^{+} \rightarrow 1^{+}$transitions, namely,

$$
\begin{aligned}
\tilde{\Omega}_{1}^{\text {recoil }}= & \lambda \frac{G_{F}^{2}}{2 \sqrt{2}} \frac{i}{\pi R^{2}} 2 \\
& \times \sum_{n, m} \phi_{n_{1}, 0}\left(r_{n}\right) \phi_{n_{2}, 0}\left(r_{m}\right) \tau_{-}(n) \tau_{-}(m) \\
& \times\left(\vec{\omega}_{n m}+\vec{\omega}_{n m}^{\prime}\right) I[x(n, m)]
\end{aligned}
$$

\footnotetext{
${ }^{1}$ It is interesting to note that in the case of neutrinoless double $\beta$ decay for the mass independent contribution one needs a correction either from the dipole term of the electron wave function or the nucleon recoil. Both are first-order corrections. The nucleon recoil term has not been extensively studied even though it was suggested by Kotani et al. long ago [14].
}

with

$$
\begin{aligned}
& \vec{\omega}_{n m}=g_{V} g_{A} \hat{r}_{n m}\left(C_{n}-C_{m}\right), \\
& \vec{\omega}_{m n}^{\prime}=g_{V}^{2} i\left(\mathbf{D}_{n}-\mathbf{D}_{m}\right) \times \hat{r}_{n, m} .
\end{aligned}
$$

(ii) One operator associated with the leptonic current:

$$
\ell_{5}=\bar{u}\left(p_{1}\right) \gamma_{5} u^{c}\left(p_{2}\right)
$$

which is suitable for $0^{+} \rightarrow 0^{+}$transitions, namely,

$$
\begin{aligned}
\tilde{\Omega}_{0}^{\text {recoil }}= & \lambda \frac{G_{F}^{2}}{2 \sqrt{2}} \frac{i}{\pi R_{0}^{2}} 2 \sum_{n, m} \phi_{n_{1}, 0}\left(r_{n}\right) \phi_{n_{2}, 0} \\
& \times\left(r_{m}\right) \tau_{-}(n) \tau_{-}(m)\left(\omega_{n m}+\omega_{n m}^{\prime}\right), \\
\omega_{n m}= & g_{V} g_{A} \hat{r}_{n m} \cdot\left[i\left(-\sigma_{n} \times D_{m}+\sigma_{m} \times D_{n}\right)\right],
\end{aligned}
$$

and

$$
\omega_{n m}^{\prime}=g_{A}^{2} \hat{r}_{n m} \cdot\left[-\sigma_{n} C_{m}+\sigma_{m} C_{n}\right] .
$$

Under the factorization approximation, one can write

$$
\begin{aligned}
& \tilde{\Omega}_{i}^{\text {recoil }}=\lambda \Lambda_{\text {eff }}\left((0 s)^{2} a_{B}^{3}\right) \Omega_{i}^{\text {recoil }}, \quad i=0,1, \\
& \Omega_{1}^{\text {recoil }}=2 \sum_{n, m} \tau_{-}(n) \tau_{-}(m)\left(\vec{\omega}_{n m}+\vec{\omega}_{n m}^{\prime}\right) f\left(x_{n, m}\right), \\
& \Omega_{0}^{\text {recoil }}=2 \sum_{n, m} \tau_{-}(n) \tau_{-}(m)\left(\omega_{n m}+\omega_{n m}^{\prime}\right) f\left(x_{n, m}\right),
\end{aligned}
$$

and then use Eqs. (17) and (18).

There remains the problem of transforming the operators $C_{n}$ and $\mathbf{D}_{n}$ into coordinate space. The operator $\mathbf{q}_{n}$ is local and leads to the operator $-i \frac{1}{R_{0}} \hat{r} \frac{d}{d x}$, where $\mathbf{x}=\mathbf{r} / R_{0}, \mathbf{r}$ being the relative distance of the two nucleons. The operator $P_{n}$ is nonlocal. Thus one finds

$$
P_{n} \rightarrow-i \frac{1}{R_{0}}\left(\overleftarrow{\nabla}_{n}-\vec{\nabla}_{n}\right),
$$

where the operator $\nabla_{n}$ operates with respect to $\mathbf{x}_{n}$ on the nuclear wave functions on the bra and ket as indicated by the arrows. As a simple example we will rewrite the operator $\hat{r}_{n m}\left(C_{n}-C_{m}\right)$ in coordinate space. We find

$$
\begin{aligned}
\hat{r}_{n m}( & \left.C_{n}-C_{m}\right) \\
= & \frac{-i}{2 M_{n} R_{0}} \hat{r}_{n m}\left\{\hat{r}_{n m} \cdot\left(\sigma_{n}-\sigma_{m}\right) \frac{d f}{d x}\right. \\
& -\left[\left(\overleftarrow{\nabla}_{n} f(x)-f(x) \vec{\nabla}_{n}\right) \cdot \sigma_{n}\right. \\
& \left.\left.-\left(\overleftarrow{\nabla}_{m} f(x)-f(x) \vec{\nabla}_{m}\right) \cdot \sigma_{m}\right]\right\} .
\end{aligned}
$$

This can be rewritten by showing the tensor character of the various components as

$$
\begin{aligned}
\hat{r}_{n m}( & \left.C_{n}-C_{m}\right) \\
= & \frac{-i}{2 M_{n} R_{0}}\left\{\left(-\frac{1}{\sqrt{3}}\left(\sigma_{n}-\sigma_{m}\right)\right.\right. \\
& \left.-\frac{2 \sqrt{2}}{\sqrt{3}}\left[\left(\hat{r}_{n m} \times \hat{r}_{n m}\right)^{2} \otimes\left(\sigma_{n}-\sigma_{m}\right)\right]\right) \frac{d f}{d x} \\
& -\frac{1}{\sqrt{3}}\left[\left(\overleftarrow{\nabla}_{n} \cdot \hat{r}_{n m} f(x)-f(x) \hat{r}_{n m} \cdot \vec{\nabla}_{n}\right) \sigma_{n}\right. \\
& \left.-\left(\overleftarrow{\nabla}_{m} \cdot \hat{r}_{n m} f(x)-f(x) \hat{r}_{n m} \cdot \vec{\nabla}_{m}\right) \sigma_{m}\right]
\end{aligned}
$$




$$
\begin{aligned}
& -\frac{2 \sqrt{2}}{\sqrt{3}}\left(\left[\left(\overleftarrow{\nabla}_{n} \otimes \hat{r}_{n m} f(x)-f(x) \hat{r}_{n m} \otimes \vec{\nabla}_{n}\right)\right]^{2} \otimes \sigma_{n}\right. \\
& \left.\left.-\left[\left(\overleftarrow{\nabla}_{m} \otimes \hat{r}_{n m} f(x)-f(x) \hat{r}_{n m} \otimes \vec{\nabla}_{m}\right)\right]^{2} \otimes \sigma_{m}\right)\right\} .
\end{aligned}
$$

In the case of the local scalar spatial contribution, it is understood that

$$
\frac{d I}{d x} \rightarrow \frac{\delta(x)}{4 \pi x^{2}}
$$

The overall tensor rank is $J=1$, the spin rank is unity, but it contains both scalar and tensor components in the orbital sector. It can thus lead to $0^{+} \rightarrow 1^{+}$transitions. The decomposition of the other operators, if needed, can be done in a similar fashion. It is quite tedious but straightforward.

The scale of the nucleon recoil contribution is set by $\left(1 / 2 m_{N} R_{0}\right)=0.07 A^{-1 / 3}$. In the case of neutrinoless double $\beta$ decay, the square of this scale should be compared with the ratio of the phase space of this $j_{L} \otimes j_{R}$ going through the electron momenta to that of the mass term.

\section{B. One of the electrons is in a $p$ orbit.}

If the resonance condition is met for such an electron, one may consider this possibility, even though the probability for finding a $p$ electron inside the nucleus is tiny. The factorization approximation should not be used in this case and the electron wave function must be part of the nuclear transition operator. The average over the nucleus of the square of the electron wave function is, however, still a good measure of the scale of the expected nuclear matrix elements. Thus in combining the above operator for the decays to negative parity states, we find that one can have transitions to $0^{+}, 1^{+}$, and $2^{+}$. The spin structure of the operator is still

$$
\omega_{0}=i \sigma_{n} \otimes \sigma_{m} \quad \text { or } \quad \omega_{1}=\sigma_{n}-\sigma_{m},
$$

i.e., antisymmetric of rank one. There appears to exist a wealth of orbital contributions.

Indeed let us assume that the $p$ electron has total angular momentum $j$ and the initial angular momentum of the atom is $J$. The structure of effective transition operator can be cast in the form

$$
\begin{aligned}
\omega_{0} \cdot \hat{r} \ell_{0} \rightarrow & U(1,1 / 2, j, 0,1 / 2,1 / 2,1,0, J) \delta_{J, 1} \\
& \times\left[\omega_{0} \cdot \hat{r} \vec{V}\right] \ell_{0}
\end{aligned}
$$

for the time component, and

$$
\begin{aligned}
\left(\omega_{1} \times \hat{r}\right) \cdot \vec{\ell} \rightarrow & \sum_{J} U(1,1 / 2, j, 0,1 / 2,1 / 2,1,0, J) \\
& \times U(1,1,0,1,1, J, J, 0, J) \\
& \times\left[\left(\omega_{1} \times \hat{r}\right) \otimes \vec{V}\right]^{J} \vec{\ell} \cdot \vec{s}
\end{aligned}
$$

for the space component $(\vec{\ell}$ is the leptonic current not to be confused with the orbital angular momentum operator). $U(\ldots)$ are the usual unitary nine- $j$ symbols needed for the recoupling of the angular momenta involved. $\vec{V}$ is essentially the two electron orbital wave function

$$
\vec{V}=\frac{1}{\sqrt{4 \pi}} \frac{\sqrt{3}}{\sqrt{4 \pi}} \frac{1}{\sqrt{2}}\left(A^{\prime \prime} \mathbf{x}_{m}+A^{\prime} \mathbf{x}_{n}\right)
$$

with

$$
\begin{aligned}
A^{\prime \prime} & =\frac{R_{0}}{r_{m}}\left(\phi_{n_{1}, 0}\left(r_{n}\right), \phi_{n_{2}, 1}\left(r_{m}\right)\right), \\
A^{\prime} & =\frac{R_{0}}{r_{n}}\left(\phi_{n_{1}, 1}\left(r_{n}\right), \phi_{n_{2}, 0}\left(r_{m}\right)\right),
\end{aligned}
$$

with $\phi_{n_{i}, \ell_{i}}, i=1,2$, the radial electron wave function. We have chosen to express the length in units of the nuclear radius $R_{0}$, i.e., $\mathbf{x}=\mathbf{r} / R_{0}$, with compensating factors in the definition of $A^{\prime}$ and $A^{\prime \prime}$, a procedure quite standard in the evaluation of the nuclear matrix element.

We found it convenient to go into the relative $\mathbf{x}=\mathbf{x}_{\mathbf{n}}-\mathbf{x}_{\mathbf{m}}$ and center of mass $2 \mathbf{X}=\mathbf{x}_{\mathbf{n}}+\mathbf{x}_{\mathbf{m}}$ coordinates and thus we get

$$
\begin{aligned}
\vec{V} & =\frac{1}{4 \pi}[A(n, m) \mathbf{x}+B(n, m) \mathbf{X}], \\
A(n, m) & =\sqrt{3} \frac{1}{\sqrt{2}} \frac{\left(A^{\prime}-A^{\prime \prime}\right)}{2}, \\
B(n, m) & =\sqrt{3} \frac{1}{\sqrt{2}}\left(A^{\prime}+A^{\prime \prime}\right) .
\end{aligned}
$$

The operator $\vec{V}$ must be symmetric under the interchange of particles $n$ and $m$ to yield a total operator which is overall symmetric under the exchange of the particles $n$ and $m$. So only the $B$ terms survive.

The next step is to perform an additional recoupling, bringing together the two spacial parts. One then finds

1. For the time component,

$$
\omega_{0} \cdot \hat{r} \ell_{0} \rightarrow \frac{B(n, m)}{4 \pi} \sum_{L} C_{0}(j, L)\left[\omega_{0} \otimes T^{L}(\hat{r}, \vec{R})\right]^{J} \delta_{J, 1} \ell_{0}
$$

with

$$
\begin{aligned}
C_{0}(3 / 2, L) & =-\frac{1}{\sqrt{2}} C_{0}(1 / 2, L) \\
& =\left(\frac{\sqrt{2}}{3 \sqrt{3}},-\frac{1}{\sqrt{6}},-\frac{\sqrt{2}}{\sqrt{15}}\right)
\end{aligned}
$$

for $L=0,1,2$, respectively, and

$$
T^{0}=\hat{r} \cdot \mathbf{X}, \quad T^{1}=\hat{r} \times \mathbf{X}, \quad T^{2}=[\hat{r} \otimes \mathbf{X}]^{2} .
$$

2. For the space component,

$$
\begin{aligned}
\omega_{1} \times \hat{r} \ell_{0} \rightarrow & \frac{B(n, m)}{4 \pi} \sum_{L, J} C_{2}(j, J, L) \\
& \times\left[\omega_{1} \otimes T^{L}(\hat{r}, \vec{R})\right]^{J} \vec{\ell} \cdot \vec{s},
\end{aligned}
$$

where the coefficients $C_{2}(j, J, L)$ are given in Table I.

By combining Eqs. (11) and (41) as well as Eqs. (13) and (43), we obtain the complete operators relevant for the decay of $0^{+}$to positive parity states with $J=0,1$, and 2 .

To simplify matters, one may seek an approximation familiar from the $0 s$ case, i.e., to take the radial part of the 
TABLE I. Coefficients $C_{2}(j, J, L)$ discussed in the text.

\begin{tabular}{cccc}
$j$ & $J$ & $L$ & $C_{2}(j, J, L)$ \\
\hline$\frac{1}{2}$ & 0 & 1 & $-\frac{1}{2}$ \\
$\frac{1}{2}$ & 1 & 0 & $-\frac{\sqrt{2}}{9}$ \\
$\frac{1}{2}$ & 1 & 1 & $-\frac{1}{2 \sqrt{6}}$ \\
$\frac{1}{2}$ & 1 & 2 & $\frac{\sqrt{\frac{5}{2}}}{3}$ \\
$\frac{3}{2}$ & 1 & 0 & $-\frac{1}{9}$ \\
$\frac{3}{2}$ & 1 & 1 & $-\frac{1}{4 \sqrt{3}}$ \\
$\frac{3}{2}$ & 1 & 2 & $\frac{\sqrt{5}}{6}$ \\
$\frac{3}{2}$ & 2 & 1 & $\frac{1}{4}$ \\
$\frac{3}{2}$ & 2 & 2 & $\frac{\sqrt{3}}{2}$ \\
\hline \hline
\end{tabular}

electron wave function outside of the integrals and replace it with its average value over the nucleus. Thus,

$$
\begin{aligned}
\tilde{\Omega}_{i} & =\lambda \Lambda_{\mathrm{eff}} \sqrt{\left\langle a_{B}^{6} B^{2}\right\rangle} \Omega_{i}, \quad \Lambda_{\mathrm{eff}}=\frac{G_{F}^{2}}{2 \sqrt{2}} \frac{1}{\pi R_{0}^{2} a_{B}^{3}}, \\
B & \leftrightarrow B(n, m),
\end{aligned}
$$

where $a_{B}$ is the Bohr radius and, again, the averaging is over the radial part alone. Thus the effective transition operators now become

$$
\begin{aligned}
\Omega_{0}= & 4 g_{A}^{2} \sum_{n, m} \tau_{-}(n) \tau_{-}(m) f\left(x_{n, m}\right) \\
& \times \sum_{L, J} C_{0}(j, L)\left[\omega_{0} \otimes T^{L}(\hat{r}, \hat{R})\right]^{J} \delta_{J, 1}, \\
\Omega_{1}= & 2 g_{V} g_{A} \sum_{n, m} \tau_{-}(n) \tau_{-}(m) f\left(x_{n, m}\right) \\
& \times \sum_{L, J} C_{2}(j, J, L)\left[\omega_{1} \otimes T^{L}(\hat{r}, \hat{R})\right]^{J} .
\end{aligned}
$$

In fact it will be adequate to consider

$$
\left\langle B^{2}\right\rangle \longrightarrow\left\langle\left(R_{n, 0}\right)^{2}\right\rangle\left\langle 3\left(\frac{R_{0}}{r} R_{n, 1}\right)^{2}\right\rangle,
$$

where $R_{n, \ell}$ are the radial parts of the bound electron wave functions. Note that the $p$ wave function involved in the averaging has been stripped of an extra power of $r$, since the $\vec{r}$ has been incorporated into the nuclear matrix element $M$.

The expression for the nuclear matrix element is the same as that of Eq. (17), where $k=J=J_{f}$ is the rank of the operator and the coupling of the two atoms is given by

$$
V^{2}=|\lambda|^{2} \Lambda_{\text {eff }}^{2}\left|a_{B}^{6} B^{2}\right\rangle|M|^{2} .
$$

We will check the factorization approximation using realistic atomic $3 s$ and $4 p_{3 / 2}$ wave functions provided to us by Shabaev [15], which were obtained by standard methods (see, e.g., Grant [16] and Bratsev et al. [17]).

Using the density profile shown in Fig. 1, corresponding to a phenomenological normalized to unity Woods-Saxon density,
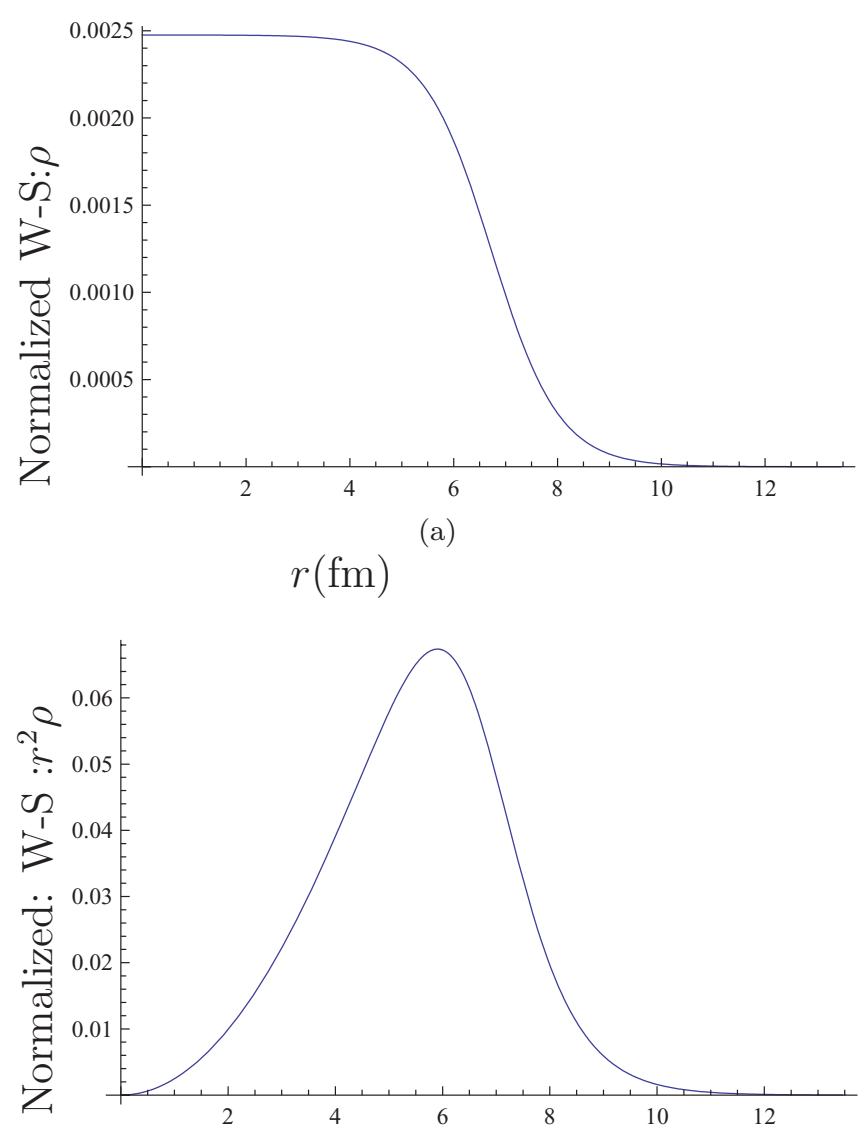

(b)

$$
r(\mathrm{fm})
$$

FIG. 1. (Color online) Normalized Woods-Saxon density profile employed in obtaining the average electron wave functions.

we find

$$
\left\langle a_{B}^{3}\left(R_{3 s}\right)^{2}\right\rangle=2.4 \times 10^{3},
$$

$$
\begin{gathered}
\left\langle 3 a_{B}^{3}\left(\frac{R_{0}}{r} R_{4 p_{3 / 2}}\right)^{2}\right\rangle=3.2 \times 10^{-2} \longrightarrow \\
\left\langle 3\left(\frac{R_{0}}{r} R_{4 p_{3 / 2}}\right)^{2}\right\rangle /\left\langle\left(R_{3 s}\right)^{2}\right\rangle=1.3 \times 10^{-5} .
\end{gathered}
$$

Using the density profile corresponding to a simplified shell model (SM) wave function shown in Fig. 2, we find

$$
\begin{gathered}
\left\langle a_{B}^{3}\left(R_{3 s}\right)^{2}\right\rangle=1.4 \times 10^{4}, \\
\left\langle 3 a_{B}^{3}\left(\frac{R_{0}}{r} R_{4 p_{3 / 2}}\right)^{2}\right\rangle=1.8 \times 10^{-1} \longrightarrow \\
\left\langle 3\left(\frac{R_{0}}{r} R_{4 p_{3 / 2}}\right)^{2}\right\rangle /\left\langle\left(R_{3 s}\right)^{2}\right\rangle=1.4 \times 10^{-5},
\end{gathered}
$$




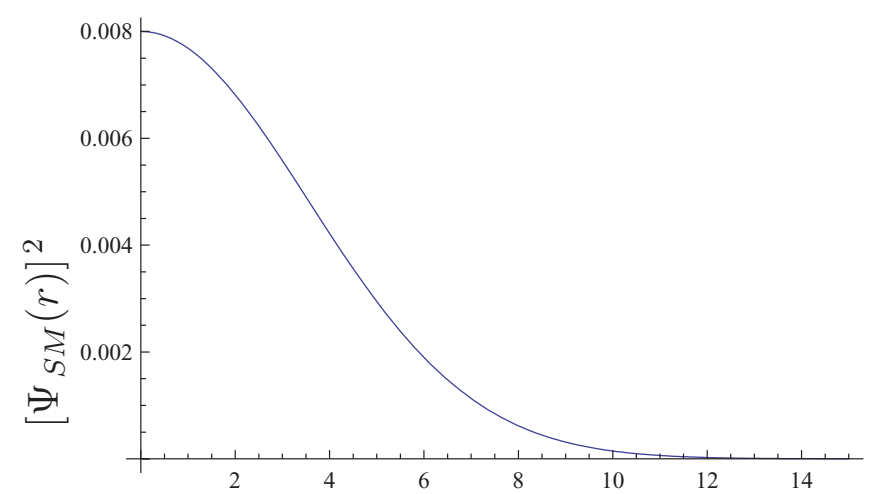

(a)

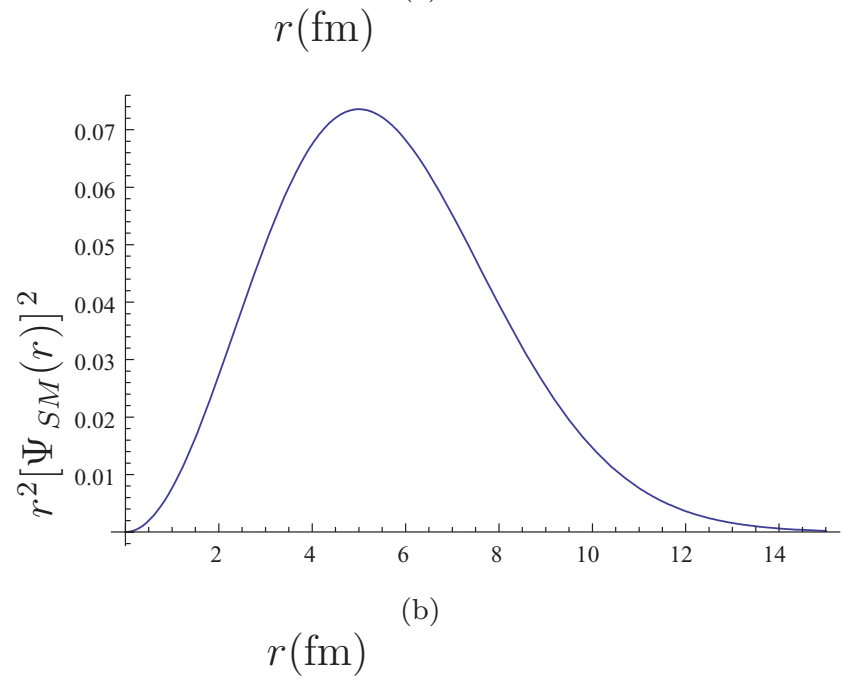

FIG. 2. (Color online) Nuclear shell model (SM) density profile employed in obtaining the average electron wave functions and checking the factorization approximation.

Had we not used the factor $3\left(R_{0} / r\right)^{2}$ the $p$ integrals would have been quite a bit smaller. The agreement in the ratio of $4 p$ to $3 s$ probabilities between the two nuclear densities is quite good. Furthermore, using a simple $0 s$ harmonic oscillator $(\mathrm{HO})$ wave function, we find that the factorization approximation works to within $10 \%$. More detailed computations using realistic and more accurate shell-model wave functions are currently underway. The results, with and without employing the factorization approximation, will be published elsewhere [18]. The nuclear matrix elements for the process ${ }^{156} \mathrm{Dy} \rightarrow{ }^{156} \mathrm{Gd}$ leading to sharp resonaces [10] $0^{-}, 1^{-}, 0^{+}, 1^{+}$, and $2^{+}$will also be calculated [18], using realistic wave functions both for the initial and the final nuclear states.

\section{DISCUSSION}

As we have mentioned, the observation of neutrinoless double decay will be a great event in particle and nuclear physics. It will establish the existence of flavor violating interactions and it will demonstrate that, independent of the dominant mechanism causing this decay, the neutrinos are Majorana particles. More importantly, it is expected to complement the great successes on neutrino oscillations by determining the elusive up to now absolute scale of neutrino mass. For this last great contribution to become unambiguous, it will be necessary to determine what fraction of this decay is due to the light neutrino mass compared to all the other competing mechanisms [3]. That is a very difficult task indeed. It may become feasible if data exist in as many targets as possible. In fact it has recently been shown that within the mass terms alone, even if both left- and right-handed currents are involved, this is, in principle, possible [19,20]. Provided, of course, that experimental data involving a sufficient number of targets become available. It will be much more difficult to achieve this if the mass independent contribution involving the $j_{L} \times j_{R}$ contribution becomes important. In this direction, the observation of double electron capture will be very useful. More specifically we have seen that (i) the neutrino mass term can proceed in neutrinoless double electron capture as in ordinary double $\beta$ decay; for this to be practical, of course, the resonance condition must be met. And (ii) neutrinoless transitions to $0^{+} \rightarrow J^{ \pm} \neq 0^{+}$can occur only if there exist right-handed interactions through the right-left interference in the leptonic sector $\left(j_{L} \otimes j_{R}\right)$. Then, in the latter case, the following is noted:

(i) The unusual transitions to the negative parity $0^{-}$and $1^{-}$ states can naturally occur in double electron capture, since they are energetically allowed if the resonance condition is met. So such an observation will unambiguously determine the neutrino mass independent lepton violating parameter, which is hard to get in neutrinoless double $\beta$ decay.

(ii) Transitions to positive parity states $\neq 0^{+}$in ordinary double $\beta$ decay can occur either through the dipole component of the electron wave functions, the most favored scenario, or if one of the hadronic currents involved contains the parity changing nucleon recoil terms.

(iii) Transitions to positive parity states with an angular momentum less than 3 in neutrinoless double electron capture can proceed (1) if one of the hadronic currents involves the recoil term, while both electrons are in $s$ states or (2) more naturally, via the negative parity operator involving ordinary nucleon currents, if one $s$ electron and one $p$ electron are involved in the capture. The probability of finding a $p$ electron in the nucleus is, however, quite small, but this disadvantage may be overcome by the collaborative effect of the expected large nuclear matrix elements and the resonance condition for capture to $2^{+}$.

(iv) The transition operators involved have a reasonable structure, but nuclear matrix elements of such operators have not yet been calculated for any system. We have no reason to expect them to be suppressed. The nuclear structure calculations involving such nuclear systems may be a true challenge to nuclear theorists.

It is clear from the above that neutrinoless double electron capture can distinguish between the mass term contribution and the neutrino mass independent $j_{L} \times j_{R}$ interference term. It can do it more efficiently than ordinary $\beta$ decay due to the possibility of the negative parity nuclear transitions. It 
cannot, of course, distinguish between the various mass terms (light neutrino, heavy neutrino, SUSY contribution, etc.), but neither can ordinary double $\beta$ decay. This can only be done by exploiting the results of at least four different experiments (leading to $0^{+}$), or a suitable combination of a sufficient number of both ground state and exited $0^{+}$state transitions. In this multiple experiment analysis, double electron capture can become a very useful partner.

[1] Goeppert-Mayer, Phys. Rev. 48, 512 (1935).

[2] W. Furry, Phys. Rev. 56, 1184 (1939).

[3] J. D. Vergados, Phys. Rep. 361, 1 (2002).

[4] F. Avignone, S. Elliott, and J. Engel, Rev. Mod. Phys. 80, 481 (2008).

[5] H. Ejiri, Phys. Rep. 338, 265 (2000).

[6] W. Rodejohann, Int. J. Mod. Phys. E 20, 1833 (2011).

[7] J. D. Vergados, Nucl. Phys. B 218, 109 (1983).

[8] J. Bernabeu, A. de Rujula, and C. Jarlskog, Nucl. Phys. B 223, 15 (1983).

[9] M. I. Krivoruchenko, F. Simkovic, D. Frekers, and A. Faessler, Nucl. Phys. A 859, 140 (2011).

[10] S. Eliseev et al., Phys. Rev. C 84, 012501(R) (2011).

[11] S. Eliseev et al., Phys. Rev. Lett. 106, 052504 (2011).

\section{ACKNOWLEDGMENTS}

The author is indebted Professor Shabaev for providing the needed atomic wave functions and to Dr. S. Eliseev and Professor Y. Novikov for discussions and useful comments. The hospitality of Professor K. Blaum in the Kernphysik, MPI-Hd and of the Theory Division at CERN are happily acknowledged.

[12] P. Belli et al., Eur. Phys. J. A 47, 91 (2011); Nucl. Phys. A 859, 126 (2011).

[13] K. Blaum, Phys. Rep. 425, 1 (2006).

[14] M. Doi, T. Kotani, H. Nishiura, K. Okuda, and E. Takasugi, Prog. Theor. Phys. 66, 1739 (1981).

[15] V. M. Shabaev and I. Tupitsin (private communication).

[16] I. Grant, Adv. Phys. 19, 747 (1970).

[17] V. Bratsev et al., Bull. Acad. Sci. USSR, Phys. Ser. 41, 173 (1977).

[18] O. Civitarese, J. Suhonen, and J. D. Vergados (unpublished).

[19] F. Simkovic, J. Vergados, and A. Faessler, Phys. Rev. D 82, 113015 (2010).

[20] A. Faessler, A. Meroni, S. T. Petcov, F. Simkovic, and J. D. Vergados, Phys. Rev. D 83, 113003 (2011). 